\title{
Universiteit
}

Leiden

The Netherlands

\section{Ultracold atoms in optical lattices}

Dickerscheid, D.B.M.; Oosten, D. van; Denteneer, P.J.H.; Stoof, H.T.C.

\section{Citation}

Dickerscheid, D. B. M., Oosten, D. van, Denteneer, P. J. H., \& Stoof, H. T. C. (2003). Ultracold atoms in optical lattices. Physical Review A, 68, 043623. doi:10.1103/PhysRevA.68.043623

Version: $\quad$ Not Applicable (or Unknown)

License: $\quad$ Leiden University Non-exclusive license

Downloaded from: https://hdl.handle.net/1887/61279

Note: To cite this publication please use the final published version (if applicable). 


\title{
Ultracold atoms in optical lattices
}

\author{
D. B. M. Dickerscheid, ${ }^{1,2}$ D. van Oosten, ${ }^{1,3}$ P. J. H. Denteneer, ${ }^{2}$ and H. T. C. Stoof ${ }^{1}$ \\ ${ }^{1}$ Institute for Theoretical Physics, University of Utrecht, Leuvenlaan 4, 3584 CE Utrecht, The Netherlands \\ ${ }^{2}$ Lorentz Institute, Leiden University, P.O. Box 9506, 2300 RA Leiden, The Netherlands \\ ${ }^{3}$ Debye Institute, University of Utrecht, Princetonplein 5, 3584 CC Utrecht, The Netherlands
}

(Received 23 June 2003; published 23 October 2003)

\begin{abstract}
Bosonic atoms trapped in an optical lattice at very low temperatures can be modeled by the Bose-Hubbard model. In this paper, we propose a slave-boson approach for dealing with the Bose-Hubbard model, which enables us to analytically describe the physics of this model at nonzero temperatures. With our approach the phase diagram for this model at nonzero temperatures can be quantified.
\end{abstract}

DOI: 10.1103/PhysRevA.68.043623

PACS number(s): 03.75.Lm, 67.40.-w, 39.25.+k

\section{INTRODUCTION}

The physics of the Bose-Hubbard model was the subject of intensive study for some years after the seminal paper by Fisher et al., which focused on the behavior of bosons in a disordered environment [1]. More recently it has been realized that the Bose-Hubbard model can also be applied to bosons trapped in so-called optical lattices [2], and meanfield theories [3-5] and exact diagonalization [6] have been successfully applied to these systems in one-, two- and threedimensional systems. The experiments performed by Greiner et al. [7] have confirmed the theoretically predicted quantum phase transition, i.e., a phase transition induced by quantum fluctuations, between a superfluid and a Mott-insulating phase. A review of the work carried out in this field has been given by Zwerger [8]. Strictly speaking, the abovementioned quantum phase transition occurs only at zero temperature [9]. At nonzero temperatures there is a "classical" phase transition, i.e., a phase transition induced by thermal fluctuations, between a superfluid phase and a normal phase and there is only a crossover between the normal phase and a Mott insulator. It is important to mention here that a Mott insulator is by definition incompressible. In principle there exists, therefore, no Mott insulator for any nonzero temperature where we always have a nonvanishing compressibility. Nevertheless, there is a region in the phase diagram where the compressibility is very close to zero and it is therefore justified to call this region for all practical purposes a Mott insulator [5]. Qualitatively this phase diagram is sketched in Fig. 1 for a fixed density. This figure shows how at a sufficiently small but nonzero temperature we start with a superfluid for small positive on-site interaction $U$, encounter a phase transition to a normal phase as the interaction strength increases, and ultimately cross over to a Mott insulator for even higher values of the interaction strength. We can also incorporate this nonzero temperature behavior into the phase diagram in Fig. 2. This figure shows how at zero temperature we only have a superfluid and a Mott insulator phase, but as the temperature is increased a normal phase appears in between these two phases.

The aim of this paper is to extend the mean-field approach for the Bose-Hubbard model to include nonzero temperature effects and make the qualitative phase diagrams in Figs. 1 and 2 more quantitative. To do that we make use of auxiliary particles that are known as slave bosons [10]. The idea behind this is that if we consider a single lattice site, the occupation number on that site can be any integer. With each different occupation number we identify a new particle. Although this means that we introduce a lot of different new particles, the advantage of this procedure is that it allows us to transform the on-site repulsion into an energy contribution that is quadratic in terms of the new particles. Because we want to be able to uniquely label each different state of the system, the new particles cannot independently be present at each lattice site. That is why we have to introduce a constraint. Using this we derive within a functional-integral formalism an effective action for the superfluid order parameter which depends on the temperature. The equivalence with previous work at zero temperature is demonstrated.

The outline of the paper is as follows. In Sec. II we introduce the slave-boson formalism and derive an effective action for the superfluid order parameter. In Sec. III we present the zero- and nonzero-temperature mean-field results. The remainder of the paper is devoted to the effect that the creation of quasiparticle-quasihole pairs has on the system.

\section{SLAVE-BOSON THEORY FOR THE BOSE-HUBBARD MODEL}

In this section we formalize the above introduced idea of the slave bosons. We rewrite the Bose-Hubbard model in terms of these slave bosons within a path-integral formulation and derive an effective action for the superfluid order parameter, which then describes all the physics of our Bose gas in the optical lattice.

The slave-boson technique was introduced by Kotliar and Ruckenstein [10], who used it to deal with the fermionic Hubbard model. A functional integral approach to the problem of hard-core bosons hopping on a lattice has been previously put forward by Ziegler [11] and Frésard [12]. Let us first shed some light on this slave-boson formalism. We consider a single site of our lattice. If the creation and annihilation operators for the bosons are denoted by $\hat{a}_{i}^{\dagger}$ and $\hat{a}_{i}$, respectively, we can form the number operator $\hat{N}_{i}=\hat{a}_{i}^{\dagger} \hat{a}_{i}$, which counts the number of bosons at the site $i$. In the slaveboson formalism, for any occupation number a pair of bosonic creation and annihilation operators is introduced that create and annihilate the state with precisely that given inte- 


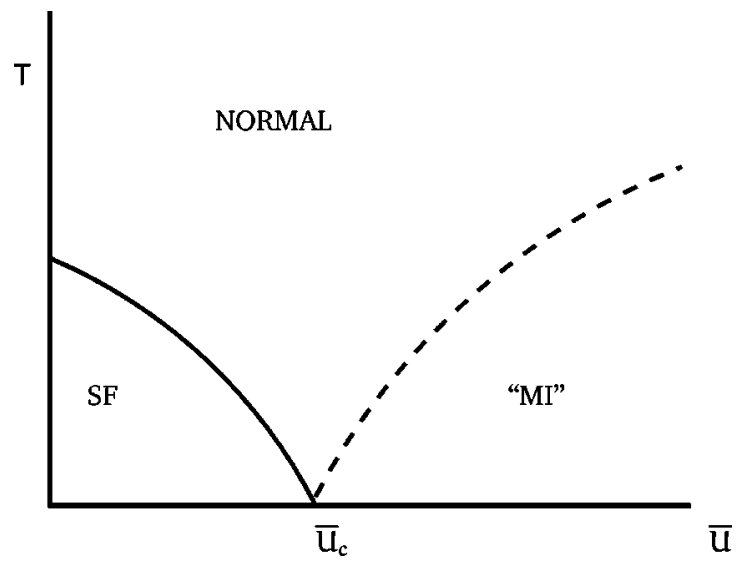

FIG. 1. Qualitative phase diagram for a fixed and integer filling fraction in terms of the temperature $T$ and the dimensionless coupling constant $\bar{U}=U / z t$, with superfluid (SF), normal, and Mott insulating phases (MI). A true Mott insulator exists only at $T=0$.

ger number of particles. The original occupation number states $\left|n_{i}\right\rangle$ are now decomposed as $\left|n_{i}^{0}, n_{i}^{1}, \ldots\right\rangle$, where $n_{i}^{\alpha}$ is the eigenvalue of the number operator $\hat{n}_{i}^{\alpha} \equiv\left(\hat{a}_{i}^{\alpha}\right)^{\dagger} \hat{a}_{i}^{\alpha}$ formed by the pair of creation $\left(\hat{a}_{i}^{\alpha}\right)^{\dagger}$ and annihilation $\hat{a}_{i}^{\alpha}$ operators that create and annihilate bosons of type $\alpha$ at the site $i$. As it stands, this decomposition is certainly not unique. For example, the original state $|2\rangle$ could be written as $|0,0,1,0, \ldots\rangle$ or as $|0,2,0, \ldots\rangle$. Our Hilbert space thus greatly increases. To make sure that every occupation occurs only once we have to introduce an additional constraint, namely,

$$
\sum_{\alpha} \hat{n}_{j}^{\alpha}=1
$$

for every site $j$. This constraint thus makes sure that there is always just one slave boson per site. Because in the positive $U$ Bose-Hubbard model bosons on the same site repel each other, high on-site occupation numbers are disfavored. It is therefore conceivable that a good approximation of the physics of the Bose-Hubbard model is obtained by allowing a relatively small maximum number, e.g., two or three or four, of bosons per site.

As is well known, the Hamiltonian of the Bose-Hubbard model reads

$$
\hat{H}=-\sum_{\langle i, j\rangle} \hat{a}_{i}^{\dagger} t_{i j} \hat{a}_{j}-\mu \sum_{i} \hat{a}_{i}^{\dagger} \hat{a}_{i}+\frac{U}{2} \sum_{i} \hat{a}_{i}^{\dagger} \hat{a}_{i}^{\dagger} \hat{a}_{i} \hat{a}_{i} .
$$

Here $\langle i, j\rangle$ denotes the sum over nearest neighbors, $t_{i j}$ are the hopping parameters, and $\mu$ is the chemical potential. Using our slave-boson operators we now rewrite Eq. (2) into the form

$$
\begin{aligned}
\hat{H}= & -\sum_{\langle i, j\rangle} \sum_{\alpha, \beta} \sqrt{\alpha+1} \sqrt{\beta+1}\left(\hat{a}_{i}^{\alpha+1}\right)^{\dagger} \hat{a}_{i}^{\alpha} t_{i j} \hat{a}_{j}^{\beta+1}\left(\hat{a}_{j}^{\beta}\right)^{\dagger} \\
& -\mu \sum_{i} \sum_{\alpha} \alpha \hat{n}_{i}^{\alpha}+\frac{U}{2} \sum_{i} \sum_{\alpha} \alpha(\alpha-1) \hat{n}_{i}^{\alpha}
\end{aligned}
$$

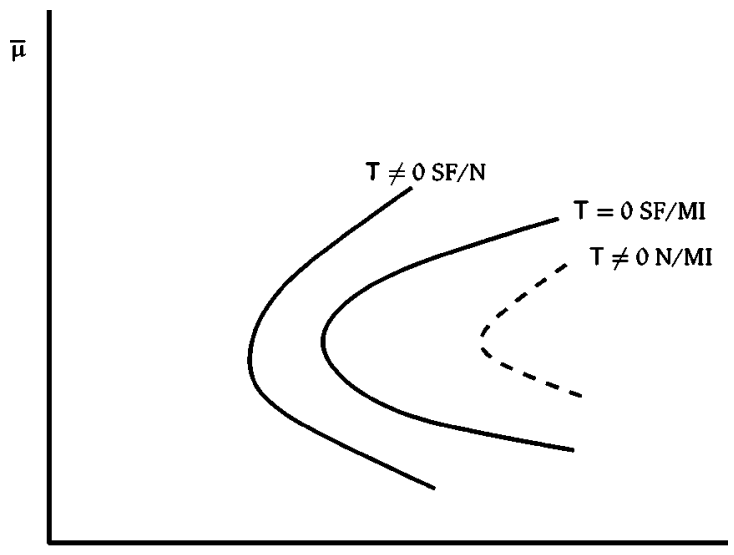

$\overline{\mathbf{u}}$

FIG. 2. Qualitative phase diagram in terms of the chemical potential $\bar{\mu}=\mu / z t$ and the dimensionless coupling constant $\bar{U}$ $=U / z t$. The solid lines indicate real phase transitions between superfluid, normal, and Mott insulating phases. the dashed line corresponds to a crossover between a normal and a Mott insulating phase.

with the additional constraint given in Eq. (1). We see that the quartic term in the original Bose-Hubbard Hamiltonian has been replaced by one that is quadratic in the slave-boson creation and annihilation operators, which is the most important motivation for the introduction of slave bosons.

Now that we have introduced the slave-boson method and derived its representation of the Bose-Hubbard model, we want to turn the Hamiltonian into an action for the imaginary time evolution. Using the standard recipe $[13,14]$ we find

$$
\begin{aligned}
S\left[\left(a^{\alpha}\right)^{*}, a^{\alpha}, \lambda\right]= & \int_{0}^{\hbar \beta} d \tau\left\{\sum_{i} \sum_{\alpha \beta}\left(a_{i}^{\alpha}\right)^{*} M^{\alpha \beta} a_{i}^{\beta}-i \sum_{i} \lambda_{i}(\tau)\right. \\
& \times\left(\sum_{\alpha} n_{i}^{\alpha}-1\right)-\sum_{\langle i, j\rangle} \sum_{\alpha, \beta} \sqrt{\alpha+1} \sqrt{\beta+1} \\
& \left.\times\left(a_{i}^{\alpha+1}\right)^{*} a_{i}^{\alpha} t_{i j} a_{j}^{\beta+1}\left(a_{j}^{\beta}\right)^{*}\right\}
\end{aligned}
$$

where $M$ is a diagonal matrix that has as the $\alpha$ th diagonal entry the term $\hbar \partial / \partial \tau-\alpha \mu+\alpha(\alpha-1) U / 2$, and $\beta=1 / k_{B} T$ is the inverse thermal energy. The real-valued constraint field $\lambda$ enters the action through,

$$
\begin{aligned}
\prod_{i} \delta\left(\sum_{\alpha} n_{i}^{\alpha}-1\right)= & \int d[\lambda] \exp \left[\frac{i}{\hbar} \int_{0}^{\hbar \beta} \sum_{i} \lambda_{i}(\tau)\right. \\
& \left.\times\left(\sum_{\alpha} n_{i}^{\alpha}-1\right) d \tau\right] .
\end{aligned}
$$

Although we have simplified the interaction term, the hopping term has become more complicated. By performing a Hubbard-Stratonovich transformation on the above action we can, however, decouple the hopping term in a similar manner as in Ref. [4]. This introduces a field $\Phi$ into the action which, as we will see, may be identified with the 
superfluid order parameter. The Hubbard-Stratonovich transformation basically consists of adding a complete square to the action, i.e., adding

$$
\begin{gathered}
\int_{0}^{\hbar \beta} d \tau \sum_{i, j}\left(\Phi_{i}^{*}-\sum_{\alpha} \sqrt{\alpha+1}\left(a_{i}^{\alpha+1}\right)^{*} a_{i}^{\alpha}\right) \\
\times t_{i j}\left(\Phi_{j}-\sum_{\alpha} \sqrt{\alpha+1} a_{j}^{\alpha+1}\left(a_{j}^{\alpha}\right)^{*}\right) .
\end{gathered}
$$

Since a complete square can be added to the action without changing the physics we see that this procedure allows us to decouple the hopping term. We also perform a Fourier transform on all fields by means of $a_{i}^{\alpha}(\tau)$ $=\left(1 / \sqrt{N_{s} \hbar \beta}\right) \sum_{\mathbf{k}, n} a_{\mathbf{k}, n}^{\alpha} e^{i\left(\mathbf{k} \cdot \mathbf{x}_{i}-\omega_{n} \tau\right)}$. If we also carry out the remaining integrals and sums we find

$$
\begin{aligned}
& S\left[\Phi^{*}, \Phi,\left(a^{\alpha}\right)^{*}, a^{\alpha}, \lambda\right] \\
& =\sum_{\mathbf{k}, n} \epsilon_{\mathbf{k}}\left|\Phi_{\mathbf{k}, n}\right|^{2}-i \frac{1}{\sqrt{N_{s} \hbar \beta}} \sum_{\mathbf{k}, \mathbf{q}} \sum_{n, n^{\prime}} \lambda_{\mathbf{q}, n^{\prime}}\left(a_{\mathbf{k}, n}^{\alpha}\right)^{*} \\
& \quad \times a_{\mathbf{k}+\mathbf{q}, n+n^{\prime}}^{\alpha}+i N_{s} \hbar \beta \lambda+\sum_{\mathbf{k}, n}\left(a_{\mathbf{k}, n}^{\alpha}\right)^{*} M^{\alpha \beta}\left(i \omega_{n}\right) a_{\mathbf{k}, n}^{\beta} \\
& \quad-\sum_{\mathbf{k}, \mathbf{k}^{\prime}, n, n^{\prime}} \frac{\epsilon_{\mathbf{k}^{\prime}}}{\sqrt{N_{s} \hbar \beta}}\left\{\left(\sum_{\alpha} \sqrt{\alpha+1}\left(a_{\mathbf{k}+\mathbf{k}^{\prime}, n+n^{\prime}}^{\alpha+1}\right)^{*} a_{\mathbf{k}, n}^{\alpha}\right)\right. \\
& \left.\quad \times \Phi_{\mathbf{k}^{\prime}, n^{\prime}}+\Phi_{\mathbf{k}^{\prime}, n^{\prime}}^{*}\left(\sum_{\alpha} \sqrt{\alpha+1} a_{\mathbf{k}+\mathbf{k}^{\prime}, n+n^{\prime}}^{\alpha+1}\left(a_{\mathbf{k}, n}^{\alpha}\right)^{*}\right)\right\},
\end{aligned}
$$

where the matrix $M\left(i \omega_{n}\right)$ is related to the matrix $M$ in Eq. (4) through a Fourier transform. Furthermore, $\lambda$ $=\left(\lambda_{\mathbf{0}, 0} / \sqrt{N_{s} \hbar \beta}\right), \epsilon_{\mathbf{k}}=2 t \sum_{j=1}^{d} \cos \left(k_{j} a\right)$, where $a$ is the lattice constant of the square lattice with $N_{s}$ lattice sites. For completeness we point out that the integration measure has become

$$
\int d\left[\left(a^{\alpha}\right)^{*}\right] d\left[a^{\alpha}\right]=\int \prod_{\mathbf{k}, n} d\left[\left(a_{\mathbf{k}, n}^{\alpha}\right)^{*}\right] d\left[a_{\mathbf{k}, n}^{\alpha}\right] \frac{1}{\hbar \beta} .
$$

In principle, Eq. (6) is still an exact rewriting of the BoseHubbard model. As a first approximation we soften the constraint by replacing the general constraint field $\lambda_{i}(\tau)$ with a time and position independent field $\lambda$. By neglecting the position dependence we enforce the constraint only on the sum of all lattice sites. Doing this we are only left with the $\lambda_{\mathbf{0}, 0}$ contribution in Eq. (6), which can then be added to the matrix $M$. The path integral over the constraint field reduces to an ordinary integral. So we have

$$
S\left[\Phi^{*}, \Phi,\left(a^{\alpha}\right)^{*}, a^{\alpha}, \lambda\right]=S_{0}+S_{I},
$$

where

$$
\begin{aligned}
S_{0} & =i N_{s} \hbar \beta \lambda+\sum_{\alpha, \beta} \sum_{\mathbf{k}, n}\left\{\epsilon_{\mathbf{k}}\left|\Phi_{\mathbf{k}, n}\right|^{2}+\left(a_{\mathbf{k}, n}^{\alpha}\right)^{*} M^{\alpha \beta}\left(i \omega_{n}\right) a_{\mathbf{k}, n}^{\beta}\right\} \\
& \equiv S_{0}^{S B}+\sum_{\mathbf{k}, n} \epsilon_{\mathbf{k}}\left|\Phi_{\mathbf{k}, n}\right|^{2} .
\end{aligned}
$$

The matrix $M^{\alpha \beta}\left(i \omega_{n}\right)=\delta_{\alpha \beta}\left[-i \hbar \omega_{n}-i \lambda-\alpha \mu+\alpha(\alpha\right.$ $-1) U / 2]$, and

$$
\begin{aligned}
S_{I}= & -\sum_{\mathbf{k}, \mathbf{k}^{\prime}, n, n^{\prime}} \frac{\epsilon_{\mathbf{k}^{\prime}}}{\sqrt{N_{s} \hbar \beta}}\left\{\left(\sum_{\alpha} \sqrt{\alpha+1}\left(a_{\mathbf{k}+\mathbf{k}^{\prime}, n+n^{\prime}}^{\alpha+1}\right) * a_{\mathbf{k}, n}^{\alpha}\right)\right. \\
& \left.\times \Phi_{\mathbf{k}^{\prime}, n^{\prime}}+\Phi_{\mathbf{k}^{\prime}, n^{\prime}}^{*}\left(\sum_{\alpha} \sqrt{\alpha+1} a_{\mathbf{k}+\mathbf{k}^{\prime}, n+n^{\prime}}^{\alpha+1}\left(a_{\mathbf{k}, n}^{\alpha}\right)^{*}\right)\right\} .
\end{aligned}
$$

The crucial idea of Landau theory is that near a critical point the quantity of most interest is the order parameter. In our theory the superfluid field $\Phi$ plays the role of the order parameter. Only $\Phi_{0,0}$ can have a nonvanishing expectation value in our case and, therefore, we can write the free energy as an expansion in powers of $\Phi_{\mathbf{0}, 0}$,

$$
F\left(\Phi_{\mathbf{0}, 0}\right)=a_{0}(\alpha, U, \mu)+a_{2}(\alpha, U, \mu)\left|\Phi_{0,0}\right|^{2}+O\left(\left|\Phi_{0,0}\right|^{4}\right),
$$

and minimize it as a function of the superfluid order parameter $\Phi_{0,0}$. We thus find that $\left\langle\Phi_{0,0}\right\rangle=0$ when $a_{2}(\alpha, U, \mu)$ $>0$ and that $\left\langle\Phi_{0,0}\right\rangle \neq 0$ when $a_{2}(\alpha, U, \mu)<0$. This means that $a_{2}(\alpha, U, \mu)=0$ signals the boundary between the superfluid and the insulator phases at zero temperature and the boundary between the superfluid and the normal phases at nonzero temperature. Therefore we are going to calculate the effective action of our theory up to second order in $\Phi$. The zeroth-order term in the expansion of the action in powers of the order parameter gives us the zeroth-order contribution $\Omega_{0}$ to the thermodynamic potential $\Omega$. We have

$$
e^{-\beta \Omega_{0}} \equiv \int \prod_{\alpha}\left(\prod_{\mathbf{k}, n} d\left[\left(a_{\mathbf{k}, n}^{\alpha}\right) *\right] d\left[a_{\mathbf{k}, n}^{\alpha}\right] \frac{1}{\hbar \beta}\right) e^{-S_{0}^{S B} / \hbar} .
$$

From this it follows that

$$
-\beta \Omega_{0}=-i N_{s} \beta \lambda+N_{s} \sum_{\alpha} \ln \left(1-e^{-\beta M^{\alpha \alpha}(0)}\right),
$$

and $M^{\alpha \alpha}(0)=[-i \lambda-\alpha \mu+\alpha(\alpha-1) U / 2]$. Next we must calculate $\left\langle S_{I}^{2}\right\rangle$ where $\langle\cdots\rangle$ denotes averaging with respect to $S_{0}$, i.e.,

$$
\begin{aligned}
\langle A\rangle= & \frac{1}{e^{-\beta \Omega_{0}}} \int \prod_{\alpha}\left(\prod_{\mathbf{k}, n} d\left[\left(a_{\mathbf{k}, n}^{\alpha}\right)^{*}\right] d\left[a_{\mathbf{k}, n}^{\alpha}\right] \frac{1}{\hbar \beta}\right) \\
& \times A\left[\left(a^{\alpha}\right)^{*}, a^{\alpha}\right] e^{-S_{0}^{S B} / \hbar} .
\end{aligned}
$$

Once we have this contribution, we automatically also find the dispersion relations for the quasiparticles in our system 
as we will see shortly. For small $\Phi$ we are allowed to expand the exponent in the integrand of the functional integral for the partition function as

$$
e^{-S / \hbar}=e^{-\left(S_{0}+S_{I}\right) / \hbar} \approx e^{-S_{0} / \hbar}\left[1-S_{I} / \hbar+\frac{1}{2}\left(S_{I} / \hbar\right)^{2}\right] .
$$

It can be shown that the expectation value of $S_{I}$ vanishes. The second-order contribution is found to be

$$
\begin{aligned}
\left\langle S_{I}^{2}\right\rangle= & 2 \sum_{\mathbf{k}, \mathbf{k}^{\prime}, n, n^{\prime}} \epsilon_{\mathbf{k}}^{2} \frac{\left|\Phi_{\mathbf{k}}\right|^{2}}{N_{s} \hbar \beta} \sum_{\alpha}(\alpha+1) \\
& \times\left\langle\left(a_{\mathbf{k}+\mathbf{k}^{\prime}, n+n^{\prime}}^{\alpha+1}\right) * a_{\mathbf{k}+\mathbf{k}^{\prime}, n+n^{\prime}}^{\alpha+1}\right\rangle\left\langle\left(a_{\mathbf{k}, n}^{\alpha}\right) * a_{\mathbf{k}, n}^{\alpha}\right\rangle .
\end{aligned}
$$

One of the sums over the Matsubara frequencies $\omega_{n}$ can be performed and the sum over $\mathbf{k}^{\prime}$ produces an overall factor $N_{s}$. We thus find

$$
\left\langle S_{I}^{2}\right\rangle=\sum_{\mathbf{k}, n} \epsilon_{\mathbf{k}}^{2} \frac{\left|\Phi_{\mathbf{k}}\right|^{2}}{\hbar \beta} \sum_{\alpha}(\alpha+1) \frac{n^{\alpha}-n^{\alpha+1}}{-i \hbar \omega_{n}-\mu+\alpha U},
$$

where we have defined the occupation numbers $n^{\alpha}$ $\equiv\left\langle\left(a_{i}^{\alpha}\right)^{*} a_{i}^{\alpha}\right\rangle$ that equal

$$
n^{\alpha}=\frac{1}{\exp \left\{\beta\left[-i \lambda-\alpha \mu+\frac{1}{2} \alpha(\alpha-1) U\right]\right\}-1} .
$$

Having performed the integrals over the slave-boson fields to second order, we can exponentiate the result to obtain the effective action for the order parameter

$$
S^{\mathrm{eff}}\left[\Phi^{*}, \Phi\right]=\left(\hbar \beta \Omega_{0}-\hbar \sum_{\mathbf{k}, n} \Phi_{\mathbf{k}, n}^{*} G^{-1}\left(\mathbf{k}, i \omega_{n}\right) \Phi_{\mathbf{k}, n}\right),
$$

where we have defined the Green's function

$$
-\hbar G^{-1}\left(\mathbf{k}, i \omega_{n}\right)=\left(\epsilon_{\mathbf{k}}-\epsilon_{\mathbf{k}}^{2} \sum_{\alpha}(\alpha+1) \frac{n^{\alpha}-n^{\alpha+1}}{-i \hbar \omega_{n}-\mu+\alpha U}\right) .
$$

This result is one of the key results of this paper, which is correct in the limit of small $\Phi_{\mathbf{k}, n}$. If we want to make the connection with the Landau theory again, we can identify the $a_{2}(\alpha, U, \mu)$ in Eq. (9) with $G^{-1}(\boldsymbol{0}, 0) / \beta$. In Sec. III we analyze this further.

\section{A. Mott insulator}

In the Mott insulator where $n_{0} \equiv\left|\left\langle\Phi_{\mathbf{0}, 0}\right\rangle\right|^{2}=0$, the thermodynamic potential is now easily calculated by integrating out the superfluid field. In detail,

$$
\begin{aligned}
Z \equiv & e^{-\beta \Omega}=\int d \lambda d\left[\Phi^{*}\right] d[\Phi] e^{-S^{\mathrm{eff} / \hbar}} \\
= & \int d \lambda \exp \left\{-\beta \Omega_{0}-\sum_{\mathbf{k}, n} \ln \left[\beta \left(\epsilon_{\mathbf{k}}-\epsilon_{\mathbf{k}}^{2}\right.\right.\right. \\
& \left.\left.\left.\times \sum_{\alpha}(\alpha+1) \frac{n^{\alpha}-n^{\alpha+1}}{-i \hbar \omega_{n}-\mu+\alpha U}\right)\right]\right\} .
\end{aligned}
$$

At this point we perform a saddle point approximation for the constraint field $\lambda$. This implies that we only take into account that value of $\lambda$ that maximizes the canonical partition function. If we now thus minimize the free energy with respect to the chemical potential and the constraint field, we get two equations that need to be solved. The first is $\partial \Omega / \partial \lambda=0$ and reads

$$
N_{s}\left(1-\sum_{\alpha} n^{\alpha}\right)-\frac{i}{\beta} \sum_{\mathbf{k}, n} G\left(\mathbf{k}, i \omega_{n}\right) \frac{\partial G^{-1}\left(\mathbf{k}, i \omega_{n}\right)}{\partial \lambda}=0 .
$$

In a mean-field approximation the last term is neglected, and this equation tells us that the sum of the average slave-boson occupation numbers must be equal to 1 . This reflects the constraint of one slave boson per site. The second equation follows from $-\partial \Omega / \partial \mu=N$ and gives

$$
N_{s} \sum_{\alpha} \alpha n^{\alpha}+\frac{1}{\beta} \sum_{\mathbf{k}, n} G\left(\mathbf{k}, i \omega_{n}\right) \frac{\partial G^{-1}\left(\mathbf{k}, i \omega_{n}\right)}{\partial \mu}=N .
$$

This equation shows how the particle density can be seen as the sum of terms $\alpha n^{\alpha}$ and a correction coming from the propagator of the superfluid order parameter. The latter is again neglected in the mean-field approximation.

\section{B. Superfluid phase}

In the superfluid phase the order parameter $\left|\Phi_{0,0}\right|^{2}$ has a nonzero expectation value. We find this expectation value by calculating the minimum of the classical part of the action, i.e., $-\hbar G^{-1}(\mathbf{0}, 0)\left|\Phi_{0,0}\right|^{2}+a_{4}\left|\Phi_{\mathbf{0}, 0}\right|^{4}$. This minimum becomes nonzero when $-\hbar G^{-1}(\mathbf{0}, 0)$ becomes negative, and is then equal to

$$
\left|\left\langle\Phi_{\mathbf{0}, 0}\right\rangle\right|^{2}=\frac{\hbar G^{-1}(\mathbf{0}, 0)}{2 a_{4}} \equiv n_{0} .
$$

In Appendix A we calculate the coefficient $a_{4}$ of the fourth-order term $\left|\Phi_{0,0}\right|^{4}$. We approximate the prefactor to the fourth-order term, which in general depends on momenta and Matsubara frequencies, with the zero-momentum and zero-frequency value of $a_{4}$ so that the approximate action to fourth order becomes 


$$
\begin{aligned}
S= & \hbar \beta \Omega_{0}-\hbar \sum_{\mathbf{k}, n} \Phi_{\mathbf{k}, n}^{*} G^{-1}\left(\mathbf{k}, i \omega_{n}\right) \Phi_{\mathbf{k}, n} \\
& +a_{4} \sum_{\mathbf{k}, \mathbf{k}^{\prime}, \mathbf{k}^{\prime \prime}} \sum_{n, n^{\prime}, n^{\prime \prime}} \Phi_{\mathbf{k}, n}^{*} \Phi_{\mathbf{k}^{\prime}, n^{\prime}}^{*} \Phi_{\mathbf{k}^{\prime \prime}, n^{\prime \prime}} \Phi_{\mathbf{k}+\mathbf{k}^{\prime}-\mathbf{k}^{\prime \prime}, n+n^{\prime}-n^{\prime \prime}}
\end{aligned}
$$

We now write the order parameter as the sum of its expectation value plus fluctuations, i.e., $\Phi_{\mathbf{0}, 0} \rightarrow \sqrt{n_{0}} \sqrt{N_{s} \hbar \beta}+\Phi_{\mathbf{0}, 0}$ and a similar expression for $\Phi_{\mathbf{0}, 0}^{*}$. If we put this into the action and only keep the terms up to second order, the contribution of the fourth-order term is given by

$$
a_{4} n_{0} \sum_{\mathbf{k}, n}\left(\Phi_{\mathbf{k}, n} \Phi_{-\mathbf{k},-n}+4 \Phi_{\mathbf{k}, n}^{*} \Phi_{\mathbf{k}, n}+\Phi_{\mathbf{k}, n}^{*} \Phi_{-\mathbf{k},-n}^{*}\right)
$$

There is also a contribution $-\hbar G^{-1}(\mathbf{0}, 0) n_{0}$ from the second-order term. To summarize, in the superfluid phase we can write the action Eq. (22) to second order as

$$
\begin{aligned}
S^{\mathrm{SF}}= & \hbar \beta \Omega_{0}-\hbar G^{-1}(\mathbf{0}, 0) n_{0} \\
& -\frac{\hbar}{2} \sum_{\mathbf{k}, n}\left(\begin{array}{ll}
\Phi_{\mathbf{k}, n}^{*} & \left.\Phi_{-\mathbf{k},-n}\right) \mathbf{G}^{-\mathbf{1}}\left(\mathbf{k}, i \omega_{n}\right)
\end{array}\right)\left(\begin{array}{c}
\Phi_{\mathbf{k}, n} \\
\Phi_{-\mathbf{k},-n}^{*}
\end{array}\right)
\end{aligned}
$$

with

$$
-\mathbf{G}^{-1}\left(\mathbf{k}, i \omega_{n}\right)=\left(\begin{array}{cc}
-G^{-1}\left(\mathbf{k}, i \omega_{n}\right)+4 \hbar a_{4} n_{0} & 2 \hbar a_{4} n_{0} \\
2 \hbar a_{4} n_{0} & -G^{-1}\left(-\mathbf{k},-i \omega_{n}\right)+4 \hbar a_{4} n_{0}
\end{array}\right) .
$$

Integrating out the field $\Phi_{\mathbf{k}, n}$ we find the Bogoliubov expression for the thermodynamic potential in the superfluid phase,

$$
\begin{aligned}
Z & \equiv e^{-\beta \Omega}=\int d \lambda d\left[\Phi^{*}\right] d[\Phi] e^{-S^{S \mathrm{~S}} / \hbar} \\
& =\int d \lambda \exp \left\{-\beta \Omega_{0}+n_{0} G^{-1}(\mathbf{0}, 0)-\operatorname{Tr}\left[\ln \left(-\hbar \beta \mathbf{G}^{-\mathbf{1}}\right)\right]\right\}
\end{aligned}
$$

\section{MEAN-FIELD THEORY}

In this section, we apply the theory we have developed in the preceding section. First, using the Landau procedure, we reproduce the mean-field zero-temperature phase diagram. We then study the phase diagram at nonzero temperatures. To do so we calculate the compressibility of our system as a function of temperature, showing how for fixed on-site repulsion $U$ the Mott insulating region gets smaller. By also looking at the condensate density as a function of temperature, we get a quantitative picture of what happens at fixed on-site repulsion $U$. The nice feature is that all our expressions are analytic. Next, we consider our system at zero temperature again and we study at the mean-field level the behavior of the compressibility as we go from the superfluid phase to the Mott insulating phase. What we find is consistent with the general idea that the quantum phase transition between the Mott insulator and the superfluid phases belongs to different universality classes depending on how you walk through the phase diagram (cf. Ref. [9]). We then obtain an analytic expression for the critical temperature of the superfluid-normal phase transition in the approximation of three slave bosons, i.e., up to doubly occupied sites. Numerically we extend this study to include a fourth slave boson and find only slight changes to $T_{c}$. From the propagator of the superfluid field we extract the dispersion relations of the quasiparticle-quasihole pairs and their temperature dependence.

\section{A. Zero-temperature phase diagram}

From the zeros of $G^{-1}(\mathbf{0}, 0)$ in Eq. (18), we obtain the mean-field phase diagram in the $(\mu, U)$ plane. For a Mott insulating state with integer filling factor $\alpha^{\prime}$ we have $n^{\alpha}$ $=\delta_{\alpha, \alpha^{\prime}}$. When this is substituted into the equation $G^{-1}(\mathbf{0}, 0)=0$ we can find the $U(\mu)$ curve that solves that equation and thus determines the size of this Mott insulating state. For given filling factor $\alpha^{\prime}$ we also define $U_{c}$ as the minimal $U$ that solves the equation. Within the Mott insulating phase we have a zero compressibility $\kappa \equiv \partial n / \partial \mu$, where

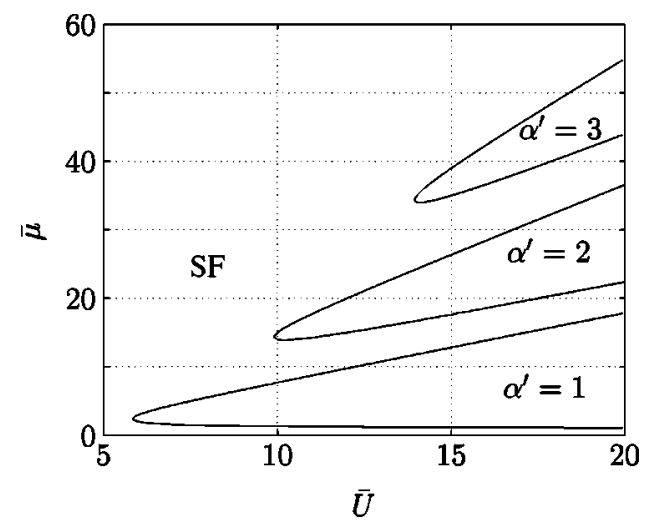

FIG. 3. Phase diagram of the Bose-Hubbard Hamiltonian as obtained from the mean-field zero-temperature limit in the slaveboson formalism. It shows the superfluid (SF) phase and the Mott insulator regions with different integer filling factors here denoted by $\alpha^{\prime}$. The vertical axis shows the dimensionless chemical potential $\bar{\mu}=\mu / z t$ and the horizontal axis shows the dimensionless interaction strength $\bar{U}=U / z t$. 

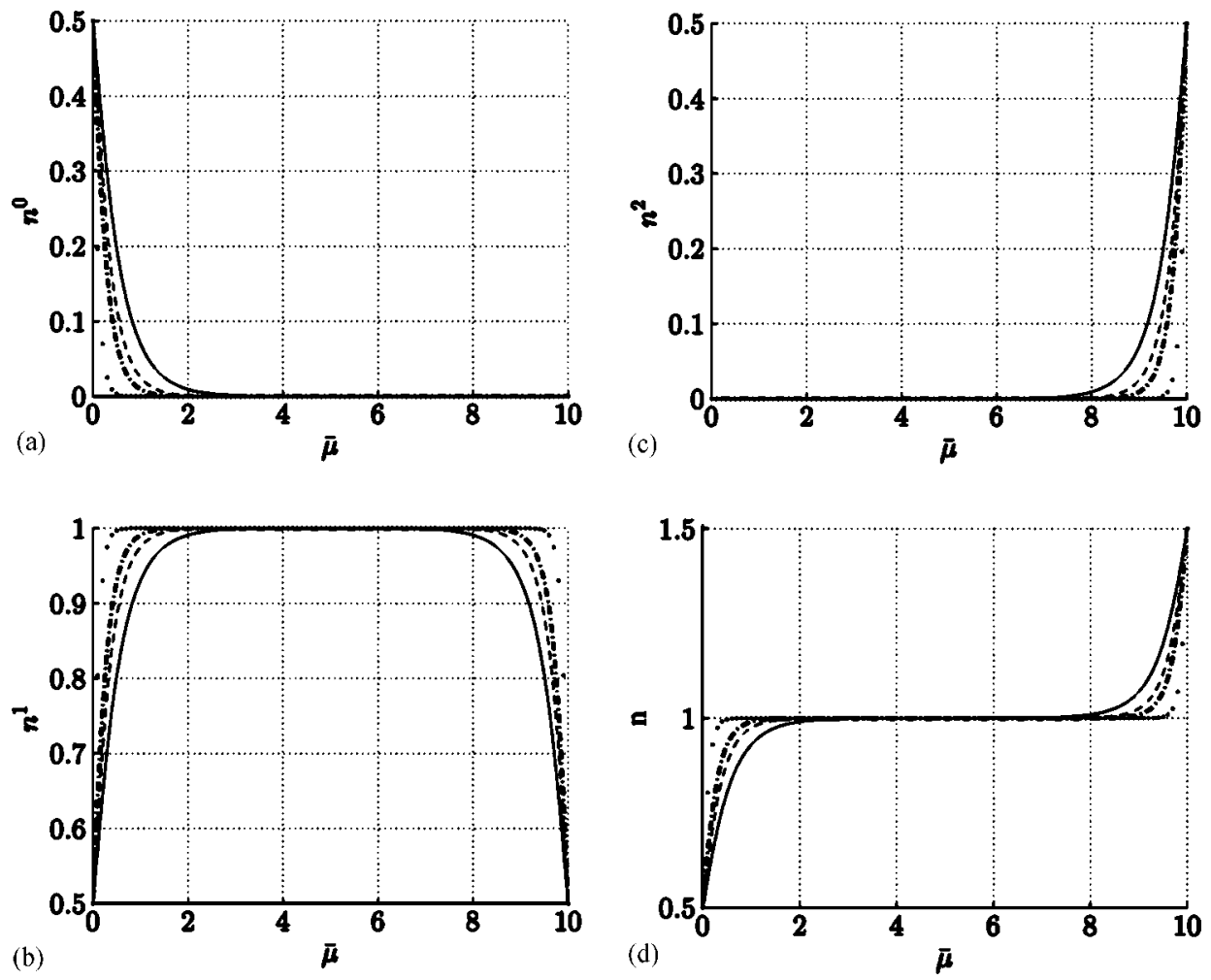

FIG. 4. Numerical solution of the slave-boson occupation numbers $n^{0}, n^{1}$, and $n^{2}$ is shown in (a)-(c) as a function of $\bar{\mu}$ for various temperatures and for fixed $U / z t=10$. (d) shows the total density $n$. The compressibility increases as a function of temperature. The solid line corresponds to $z t \beta=2$, the dashed line corresponds to $z t \beta=3$, the dasheddotted line corresponds to zt $\beta$ $=4$, and the dotted line corresponds to $z t \beta=10$. $n=n(\mu, U)$ is the total density as determined from the thermodynamic potential. Straightforward calculation gives that we are in a Mott insulating phase whenever $\bar{\mu}$ lies between $\bar{\mu}_{-}^{\alpha^{\prime}}$, and $\bar{\mu}_{+}^{\alpha^{\prime}}$ where

$$
\bar{\mu}_{ \pm}^{\alpha^{\prime}}=\frac{1}{2}\left[\bar{U}\left(2 \alpha^{\prime}-1\right)-1\right] \pm \frac{1}{2} \sqrt{\bar{U}^{2}-2 \bar{U}\left(2 \alpha^{\prime}+1\right)+1} .
$$

Here we have introduced the dimensionless chemical potential $\bar{\mu} \equiv \mu / z t$ and on-site repulsion strength $\bar{U} \equiv U / z t$. When $\bar{\mu}$ does not lie between any $\bar{\mu}_{-}^{\alpha^{\prime}}$ and $\bar{\mu}_{+}^{\alpha^{\prime}}$ the "superfluid" density $\left|\left\langle\Phi_{\mathbf{0}, 0}\right\rangle\right|^{2}$ will no longer be zero and the Mott insulating phase has disappeared. We have drawn the zerotemperature phase diagram in Fig. 3. Our slave-boson approach reproduces here the results of previous mean-field studies $[1,3,4]$. For nonzero temperatures the equation $G^{-1}(\mathbf{0}, 0)=0$ no longer describes a quantum phase transition between a superfluid and a Mott insulator but it describes a thermal phase transition between a superfluid and a normal phase. We will look into this in more detail in Sec. III F.

\section{B. Compressibility}

To see what happens to the Mott insulator as we move away from zero temperature we must look at the compressibility as a function of temperature. Numerically we have solved Eq. (20), which gives us the occupation numbers of the slave bosons as depicted in Fig. 4. With that we can determine the total density in the phase where the order parameter is zero. It is clear that within a mean-field approximation the compressibility at zero temperature is exactly zero. In Fig. 4 we have plotted the total density as a function of temperature. As the temperature is raised we find that the compressibility, which is the slope of the curve, for a given value of $\bar{U}$ becomes nonzero for all values of $\bar{\mu}$. Although the slope can be exponentially small, this shows that there is no longer a Mott insulator present. Because we are dealing with a crossover there is no unique way to define the transition from a normal to a Mott insulator phase. There are various ways to determine the crossover line. For instance, we can define it by requiring that $\Delta(T) / k_{B} T$ is of order 1 , where $\Delta(T)$ is defined as the difference of the quasiparticle and quasihole dispersions at $\mathbf{k}=0$. Another possibility is to define it by requiring that the incommensurability is equal to a certain small value.

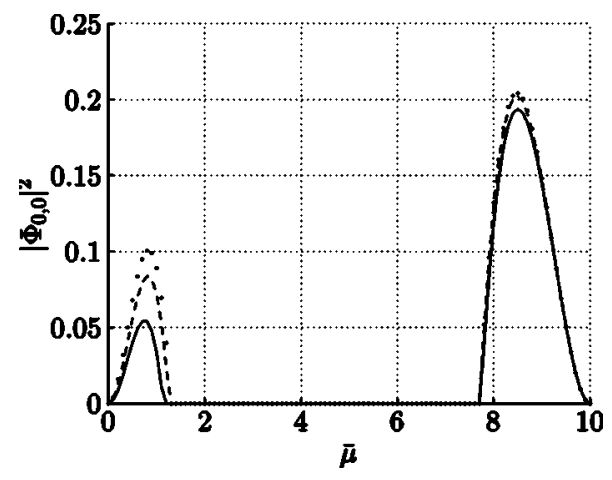

FIG. 5. Superfluid density $\left|\Phi_{0,0}\right|^{2}$ as a function of $\bar{\mu}$ for various temperatures and for $U / z t=10$. The superfluid density as well as the region of superfluid phase diminish as a function of increasing temperature. The vanishing of $\left|\Phi_{0,0}\right|^{2}$ at $\bar{\mu}=0$ and $\bar{\mu}=10$ is an artefact of our approximation (see text). In the figure the dotted line corresponds to zt $\beta=10$, the dashed line corresponds to zt $\beta=3$, and the solid line corresponds to $z$ t $\beta=2$. 


\section{Superfluid density}

In a mean-field approximation the superfluid density is extracted from the action by finding the $\left|\left\langle\Phi_{0,0}\right\rangle\right|^{2}$ that minimizes the fourth-order action in Eq. (22),

$$
\left|\left\langle\Phi_{\mathbf{0}, 0}\right\rangle\right|^{2}=\frac{\hbar G^{-1}(\mathbf{0}, 0)}{2 a_{4}},
$$

whenever $\mu$ is not between $\mu_{-}^{\alpha^{\prime}}$ and $\mu_{+}^{\alpha^{\prime}}$, and zero otherwise. We have plotted this expectation value in Fig. 5 for $\alpha^{\prime}=1$. In this figure we see how the superfluid density grows as a function of $\mu$ moving away from the Mott insulator phase. Our expansion of the Landau free energy is only valid around the edge of the Mott lobes and therefore breaks down when we go too far away from the Mott insulator. This can be seen in the figure as the decrease of the superfluid density when $\mu$ approaches 0 and/or $U$. It can also be seen from the propagator of the superfluid field, which has poles when $\mu=\alpha U$. For $\mu$ not too far away from the insulating phase the figure quantitatively agrees with the ones calculated by other authors [4].

\section{Bogoliubov dispersion relation}

We now demonstrate that the dispersion $\hbar \omega_{\mathbf{k}}$ is linear in $\mathbf{k}$ in the superfluid phase and that the spectrum is gapless. In the superfluid phase we can expand around the expectation value $n_{0}=\hbar G^{-1}(\mathbf{0}, 0) / 2 a_{4}$ of the order parameter. Up to quadratic order this gives

$$
\begin{aligned}
S= & \hbar \beta \Omega_{0}-\hbar \sum_{\mathbf{k}, n} \Phi_{\mathbf{k}, n}^{*} G^{-1}\left(\mathbf{k}, i \omega_{n}\right) \Phi_{\mathbf{k}, n} \\
& +a_{4} n_{0} \sum_{\mathbf{k}, n}\left(\Phi_{\mathbf{k}, n} \Phi_{-\mathbf{k},-n}+4 \Phi_{\mathbf{k}, n}^{*} \Phi_{\mathbf{k}, n}+\Phi_{\mathbf{k}, n}^{*} \Phi_{-\mathbf{k},-n}^{*}\right) .
\end{aligned}
$$

From this we find the dispersion relation $\hbar \omega_{\mathbf{k}}$ in the superfluid in the usual way. We perform an analytic continuation $G^{-1}\left(\mathbf{k}, i \omega_{n}\right) \rightarrow G^{-1}\left(\mathbf{k}, \omega_{k}\right)$ and find

$$
\hbar \omega_{\mathbf{k}}=\hbar \sqrt{\left[G^{-1}\left(\mathbf{k}, \omega_{\mathbf{k}}\right) / 2-G^{-1}(\mathbf{0}, 0)\right]^{2}-\left[G^{-1}(\mathbf{0}, 0) / 2\right]^{2}} .
$$

Note that $\left(\mathbf{k}, \omega_{\mathbf{k}}\right)=(\mathbf{0}, 0)$ is a solution. Expanding around this solution in $\mathbf{k}$ now gives

$$
\hbar \omega_{\mathbf{k}}=a \frac{\hbar G^{-1}(\mathbf{0}, 0)}{\sqrt{2}}|\mathbf{k}|
$$

where $a$ is again the lattice constant.

\section{E. Near the edges of the Mott lobe}

If we substitute the vacuum expectation value of the order parameter back into our effective action, we see that the zeroth-order contribution to the thermodynamic potential in the superfluid phase in mean-field approximation is given by

$$
\hbar \beta \Omega=\hbar \beta \Omega_{0}-\frac{\left[\hbar G^{-1}(\mathbf{0}, 0)\right]^{2}}{2 a_{4}} .
$$

From this the particle density can be obtained by making use of the thermodynamic identity $N=-\partial \Omega / \partial \mu$. We can calculate this at $T=0$ and take the limit $\mu \rightarrow \mu_{ \pm}^{\alpha^{\prime}}$ to show that the derivative of the density with respect to $\mu$, i.e, $\partial n / \partial \mu$ shows a kink for all $U \neq U_{c}$. This means that only if we walk through the tip of the Mott lobes there is not a kink in the compressibility. In fact, it is not hard to see why this is true. At zero temperature the roots of $-\hbar G^{-1}(\mathbf{0}, 0)$ are by definition $\mu_{ \pm}^{\alpha^{\prime}}$. This means that we can write $-\hbar G^{-1}(\mathbf{0}, 0)$ $=C\left(\mu-\mu_{-}^{\alpha^{\prime}}\right)\left(\mu-\mu_{+}^{\alpha^{\prime}}\right)$. The proportionality constant can be shown to be equal to $C=\epsilon_{0}\left\{\left(\alpha^{\prime} U-\mu\right)\left[\left(\alpha^{\prime}-1\right) U-\mu\right]\right\}$. This then shows that the thermodynamic potential is

$$
\hbar \beta \Omega=\hbar \beta \Omega_{0}+\frac{C^{2}}{4} \frac{\left(\mu-\mu_{-}^{\alpha^{\prime}}\right)^{2}\left(\mu-\mu_{+}^{\alpha^{\prime}}\right)^{2}}{a_{4}} .
$$

Remembering that the density is the derivative of the thermodynamic potential we see that the second derivative of the thermodynamic potential with respect to $\mu$ can show a nonzero value upon approaching the Mott lobe. Since in the Mott isolator the density is constant and equal to $\alpha^{\prime}$ we have shown the existence of a kink in the slope of the density for all paths not going through the tip of the Mott lobe. This causes the difference in the universality class of the quantum phase transition.

\section{F. The superfluid-normal phase transition}

In this subsection, we show that it is possible to obtain an analytical expression for the critical temperature $T_{\mathrm{c}}$ of the transition between superfluid and normal phases as a function of $U$, for values of $U$ below the critical $U$ of the zerotemperature superfluid-Mott insulator transition. The analytical result is obtained if we include occupations up to two per site, i.e., three slave bosons or occupation numbers $n^{0}, n^{1}$, and $n^{2}$. Along similar lines, $T_{\mathrm{c}}$ can be found numerically if more slave bosons are included. We have carried out this procedure for the case of adding a fourth boson (triple occupancy) and find only modest quantitative changes.

If we restrict the system to occupancies 0,1 , and 2 , and fix the total density $n \equiv N / N_{s}$ at 1 , the occupation numbers $n^{0}, n^{1}$, and $n^{2}$ should obey the following relations if we neglect fluctuation corrections [cf. Eq. (20)]:

$$
n^{0}+n^{1}+n^{2}=1
$$

and

$$
n^{1}+2 n^{2}=1 \text {. }
$$

The $n^{\alpha}$ are furthermore given by Eq. (16), enabling us to eliminate $\lambda$ and express $n^{0}$ and $n^{2}$ in terms of $n^{1}$. We obtain

$$
n^{0}=\frac{n^{1}}{\left(n^{1}+1\right) \exp (\beta \mu)-n^{1}}
$$




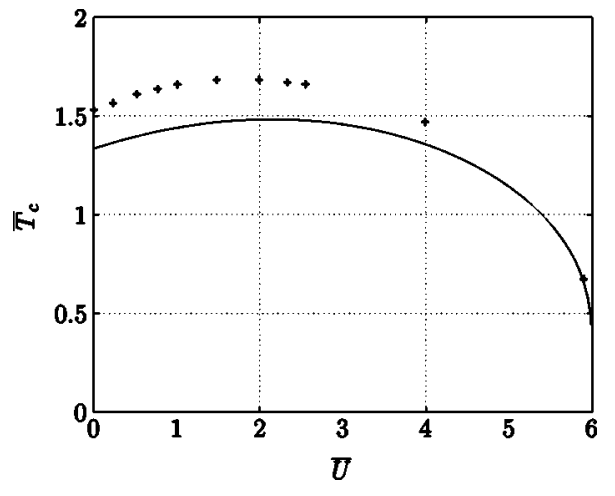

FIG. 6. Critical temperature $T_{c}$ of the superfluid-normal phase transition as a function of the interaction strength $\bar{U}=U / z t$. The solid line is an analytic expression obtained in the approximation where we only take into account three slave bosons. The pluses correspond to a numerical solution for the case of four slave bosons.

and

$$
n^{2}=\frac{n^{1}}{\left(n^{1}+1\right) \exp [\beta(U-\mu)]-n^{1}} .
$$

The constraints in Eqs. (33) and (34) immediately lead to $n^{0}=n^{2}$, so that, according to Eqs. (35) and (36), we must have $\mu=U / 2$. We notice that at this level of approximation, we obtain a slight discrepancy with the result from Sec. III A that at zero temperature the critical value of $\bar{U}$ of the superfluid-Mott insulator transition, which is the limiting $\bar{U}$ for the superfluid-normal transition that is addressed here, is according to Eq. (26) with $\alpha^{\prime}=1$ determined by $\bar{\mu}=(\bar{U}$ $-1) / 2[15]$.

As argued above, the criticality condition for the superfluid-normal transition is obtained by putting $G^{-1}(\mathbf{0}, 0)=0$. Restricting the sum in the right-hand side of Eq. (18) to $\alpha=0$ and $\alpha=1$, we obtain [16]

$$
1=\frac{2}{\bar{\mu}-\bar{U}}\left(n^{2}-n^{1}\right)+\frac{1}{\bar{\mu}}\left(n^{1}-n^{0}\right) .
$$

Since the relation between $\mu$ and $U$ is fixed by Eqs. (33) and (34), and $n^{0}$ and $n^{2}$ can be expressed in $n^{1}$ as $n^{0}=n^{2}=(1$ $\left.-n^{1}\right) / 2$, the criticality condition Eq. (37) results in a remarkably simple relation between $n^{1}$ and $\bar{U}$ at $T_{\mathrm{c}}$, namely, $n^{1}$ $=(\bar{U}+3) / 9$. Using this in Eq. (35) leads to the following analytic formula for $\bar{T}_{\mathrm{c}} \equiv T_{c} / z t$ for the superfluid-normal transition:

$$
k_{\mathrm{B}} \bar{T}_{\mathrm{c}}=\frac{\bar{U}}{2} \ln ^{-1}\left[\frac{(\bar{U}-24)(\bar{U}+3)}{(\bar{U}-6)(\bar{U}+12)}\right] \text {. }
$$

It is straightforward to generalize this procedure to arbitrary integer density $\alpha^{\prime}$ while allowing occupation numbers $n^{\alpha^{\prime}-1}, n^{\alpha^{\prime}}, n^{\alpha^{\prime}+1}$ only. The result is

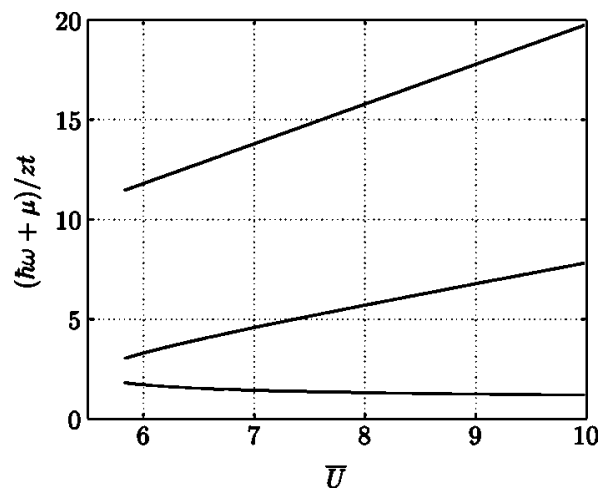

FIG. 7. The dispersion relations for $\mathbf{k}=0$ in the case where we take into account higher filling factors at nonzero temperature. On the vertical axis is $(\hbar \omega+\mu) / z t$ and on the horizontal axis is $\bar{U}$. Here we have taken into account all the terms with $\alpha=0,1,2$ at a temperature of $z$ t $\beta=10$.

$$
k_{\mathrm{B}} \bar{T}_{\mathrm{c}}^{\alpha^{\prime}}=\frac{\bar{U}}{2} \ln ^{-1}\left[\frac{\left[\bar{U}-8\left(2 \alpha^{\prime}+1\right)\right]\left[\bar{U}+\left(2 \alpha^{\prime}+1\right)\right]}{\left[\bar{U}-2\left(2 \alpha^{\prime}+1\right)\right]\left[\bar{U}+4\left(2 \alpha^{\prime}+1\right)\right]}\right] \text {. }
$$

The critical temperature $T_{\mathrm{c}}$ for integer filling factor $n$ $\equiv N / N_{s}=1$, i.e., Eq. (38), is plotted in Fig. 6. The overall qualitative behavior is as one would expect (cf. Fig. 1). A few finer details appear to be less satisfactory. For instance, $T_{\text {c }}$ vanishes for $\bar{U}=6$, whereas we would expect this to coincide with the mean-field result for $\bar{U}_{\mathrm{c}}$ for the superfluidMott insulator transition for the first Mott lobe, i.e., $\bar{U}_{\mathrm{c}}$ $=5.83$ obtained from Eq. (26) with $\alpha^{\prime}=1$. We note that the discrepancy is not large and is even smaller for the higher Mott lobes. Indeed $\bar{U}\left(T_{\mathrm{c}} \rightarrow 0\right)=2\left(2 \alpha^{\prime}+1\right)$ versus $\bar{U}_{\mathrm{c}}$ $=\left(2 \alpha^{\prime}+1\right)+\sqrt{\left(2 \alpha^{\prime}+1\right)^{2}-1}$. Another feature is the maximum in the $\bar{T}_{\mathrm{c}}(U)$ curve (cf. Fig. 1 and Ref. [3]). Both features mentioned are caused by the fact that the two conditions Eqs. (33) and (34) are strictly enforced, whereas they become less appropriate for small $U$. The exact solution [17] for four slave bosons on a four site lattice for small $\bar{U}$ shows that a better result may be obtained if a fourth boson occupation number $n^{3}$ is included in our approach. The set of equations to be solved then becomes, again for $n=1$,

$$
\begin{gathered}
n^{0}+n^{1}+n^{2}+n^{3}=1, \\
n^{1}+2 n^{2}+3 n^{3}=1, \\
\frac{3}{\bar{\mu}-2 \bar{U}}\left(n^{3}-n^{2}\right)+\frac{2}{\bar{\mu}-\bar{U}}\left(n^{2}-n^{1}\right)+\frac{1}{\bar{\mu}}\left(n^{1}-n^{0}\right)=1 .
\end{gathered}
$$

Again $n^{0}, n^{2}$, and $n^{3}$ can easily be expressed in terms of $n^{1}$, but no exact solution appears to be possible in this case. However, we have managed to find solutions numerically. The results for $T_{\mathrm{c}}$ are depicted in Fig. 6 and show fairly little quantitative change compared to the analytical result Eq. (38). In particular, $\bar{T}_{\mathrm{c}}$ still vanishes for $\bar{U} \approx 6$, and the maxi- 
mum is still there, although shifted to a lower $\bar{U} \approx 1.8 \mathrm{com}$ pared to $\bar{U}=2.15$ for Eq. (38). It is satisfactory to find that for the higher values of $\bar{U}, n^{1}$ starts to increase rapidly towards 1, signaling the approach of the Mott-insulator phase, whereas $n^{3}$ is almost negligible $(<1 \%)$ already for $\bar{U} \approx 3$, supporting a description in terms of three slave bosons only [18].

\section{G. Quasiparticle-quasihole dispersion relations}

Consider now the propagator $G^{-1}(\mathbf{k}, \omega)$, given by

$$
-\hbar G^{-1}(\mathbf{k}, \omega)=\left(\epsilon_{\mathbf{k}}-\epsilon_{\mathbf{k}}^{2} \sum_{\alpha}(\alpha+1) \frac{n^{\alpha}-n^{\alpha+1}}{-\hbar \omega-\mu+\alpha U}\right) .
$$

At zero temperature and for a given integer filling factor $\alpha^{\prime}$, we have in a mean-field approximation that $n^{\alpha}=\delta_{\alpha, \alpha^{\prime}}$ and we retrieve the previously found result for the quasiparticlequasihole dispersions [4]. In this case the real solutions of $\hbar \omega$ follow from a quadratic equation $G^{-1}\left(\mathbf{k}, \omega_{n}\right)=0$. At nonzero temperature the occupation numbers, in general, are all nonzero and there will be more than just two solutions for $\hbar \omega$. In the set of solutions there are still two solutions that correspond to the original single quasiparticle and quasihole dispersions. The physical interpretation of the other solutions is that they correspond to the excitation of a higher number of quasiparticles and quasiholes. In Fig. 7. we show the three low-lying excitation energies for $\mathbf{k}=0$ at a temperature of $z t \beta=10$. To obtain an analytic expression for the single quasiparticle-quasihole dispersion we only take into account the two terms in the sum in Eq. (18) which have numerators $n^{\alpha^{\prime}-1}-n^{\alpha^{\prime}}$ and $n^{\alpha^{\prime}}-n^{\alpha^{\prime}+1}$. These correspond to processes where the occupation of a site changes between $\alpha^{\prime}-1, \alpha^{\prime}$, and $\alpha^{\prime}+1$. We find

$$
\begin{aligned}
\hbar \omega_{\mathbf{k}}^{q p, q h}= & -\mu+\frac{U}{2}+\frac{1}{2} \epsilon_{\mathbf{k}}\left[\alpha^{\prime} n^{\alpha^{\prime}-1}-n^{\alpha^{\prime}}+\left(\alpha^{\prime}+1\right) n^{\alpha^{\prime}+1}\right] \\
& \pm \frac{1}{2} \sqrt{U^{2}+2\left[\alpha^{\prime} n^{\alpha^{\prime}-1}-\left(1+2 \alpha^{\prime}\right) n^{\alpha^{\prime}}+\left(1+\alpha^{\prime}\right) n^{\alpha^{\prime}+1}\right] U \epsilon_{\mathbf{k}}+\left[\alpha n^{\alpha^{\prime}-1}+n^{\alpha^{\prime}}-\left(1+\alpha^{\prime}\right) n^{\alpha^{\prime}+1}\right]^{2} \epsilon_{\mathbf{k}}^{2}} .
\end{aligned}
$$

In Fig. 8 we have plotted these dispersions at $\mathbf{k}=\mathbf{0}$ as a function of $U$. Comparison with Fig. 7 shows that Eq. (44) gives an appropriate description of the single quasiparticlequasihole dispersions. As can be seen from Fig. 8, the tip of the lobe moves to smaller $U$ as a function of increasing temperature. This can be understood because that point now describes the superfluid-normal phase transition (cf. Figs. 1 and 6). In Fig. 9 we show how the superfluid-normal boundary in the $\bar{\mu}-\bar{U}$ plane evolves for nonzero temperatures. If we define the gap as the difference between the two solutions at $\mathbf{k}=0$, we find that the gap grows bigger as the temperature increases. As we have seen in Sec. III B it is incorrect, however, to conclude from this that the region of the Mott insulating phase in the $\mu-U$ phase diagram grows as temperature increases. As mentioned previously, strictly speaking there is no Mott insulator away from zero temperature and at nonzero temperatures there is only a crossover between a phase which has a very small compressibility and the normal phase.

\section{FLUCTUATIONS}

In this section we make a first step towards the study of fluctuation effects and derive an identity between the atomic Green's function and the superfluid Green's function in Eq. (18). This we then use to calculate the atomic particle density. In Appendix B we show that the easiest way to calculate the density is by making use of currents that couple to the atomic fields. We start with the action of the Bose-Hubbard model

$$
\begin{aligned}
S\left[a^{*}, a\right]= & \int_{0}^{\hbar \beta} d \tau\left[\sum_{i} a_{i}^{*}\left(\hbar \frac{\partial}{\partial \tau}-\mu\right) a_{i}-\sum_{i j} t_{i j} a_{i}^{*} a_{j}\right. \\
& \left.+\frac{U}{2} \sum_{i} a_{i}^{*} a_{i}^{*} a_{i} a_{i}\right]
\end{aligned}
$$

We are interested in calculating the $\left\langle a_{i}^{*} a_{i}\right\rangle$ correlation function. Therefore we add currents $J^{*}, J$ that couple to the $a^{*}$ and $a$ fields as

$$
\begin{aligned}
Z\left[J^{*}, J\right]= & \int d\left[a^{*}\right] d[a] \exp \left\{-S_{0} / \hbar+\frac{1}{\hbar} \int_{0}^{\hbar \beta} d \tau \sum_{i j} a_{i}^{*} t_{i j} a_{j}\right. \\
& \left.+\int_{0}^{\hbar \beta} d \tau \sum_{i}\left[J_{i}^{*} a_{i}+a_{i}^{*} J_{i}\right]\right\}
\end{aligned}
$$

Here $S_{0}=S_{0}\left[a^{*}, a\right]$ denotes the action for $t_{i j}=0$. The most important step in the remainder of the calculation is to a perform again a Hubbard-Stratonovich transformation by adding a complete square to the action. The latter can be written as

$$
\int d \tau \sum_{i, j}\left(a_{i}^{*}-\Phi_{i}^{*}+\hbar t_{i j^{\prime}}^{-1} J_{j^{\prime}}^{*}\right) t_{i j}\left(a_{j}-\Phi_{j}+\hbar t_{j j^{\prime \prime}}^{-1} J_{j^{\prime \prime}}\right),
$$




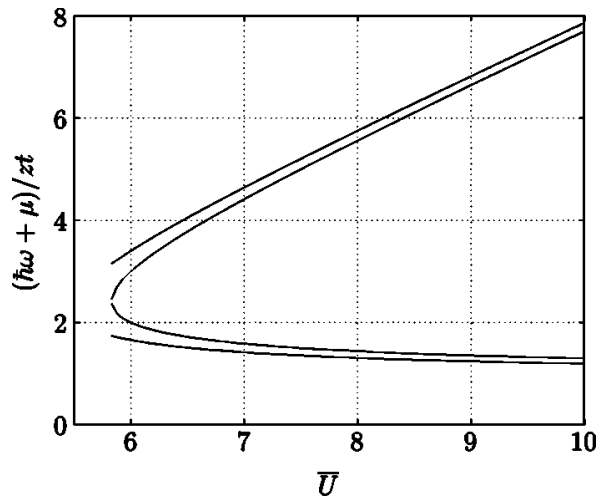

FIG. 8. Dispersion relations $\hbar \omega+\mu$ as a function of $U / z t$ for $\mathbf{k}=\mathbf{0}$ for zero and nonzero temperatures. The inner lobe corresponds to zero temperature. The outer lobe corresponds to a temperature of zt $\beta=3$. Here we have only taken into account the first three terms in the right-hand side of Eq. (18), i.e., in the sum we only include the terms with $\alpha=0$ and $\alpha=1$.

where the sums over $j^{\prime}$ and $j^{\prime \prime}$ are left implicit for simplicity. Straightforward algebra yields

$$
\begin{aligned}
Z\left[J^{*}, J\right]= & \int d\left[\Phi^{*}\right] d[\Phi] \exp \left\{\sum _ { \mathbf { k } , n } \left(-\hbar \Phi_{\mathbf{k}, n}^{*} G^{-1}\left(\mathbf{k}, i \omega_{n}\right)\right.\right. \\
& \left.\left.\times \Phi_{\mathbf{k}, n}+J_{\mathbf{k}, n}^{*} \Phi_{\mathbf{k}, n}+J_{\mathbf{k}, n} \Phi_{\mathbf{k}, n}^{*}-\frac{\hbar}{\epsilon_{\mathbf{k}}} J_{\mathbf{k}, n}^{*} J_{\mathbf{k}, n}\right)\right\} .
\end{aligned}
$$

Differentiating twice with respect to the currents gives then the relation

$$
\begin{array}{r}
\left.\frac{1}{Z[0,0]} \frac{\delta^{2}}{\delta J_{\mathbf{k}, n}^{*} \delta J_{\mathbf{k}, n}} Z\left[J^{*}, J\right]\right|_{J^{*}, J=0} \\
=\left\langle a_{\mathbf{k}, n}^{*} a_{\mathbf{k}, n}\right\rangle=\left\langle\Phi_{\mathbf{k}, n}^{*} \Phi_{\mathbf{k}, n}\right\rangle-\frac{\hbar}{\epsilon_{\mathbf{k}}}
\end{array}
$$

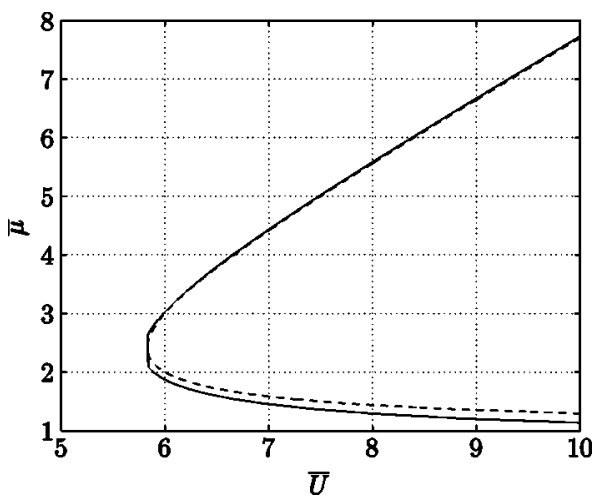

FIG. 9. The $\bar{\mu}-\bar{U}$ phase diagram for zero and nonzero temperatures. The inner lobe corresponds to the zero-temperature case. The outer lobe corresponds to a temperature of $z t \beta=2$.

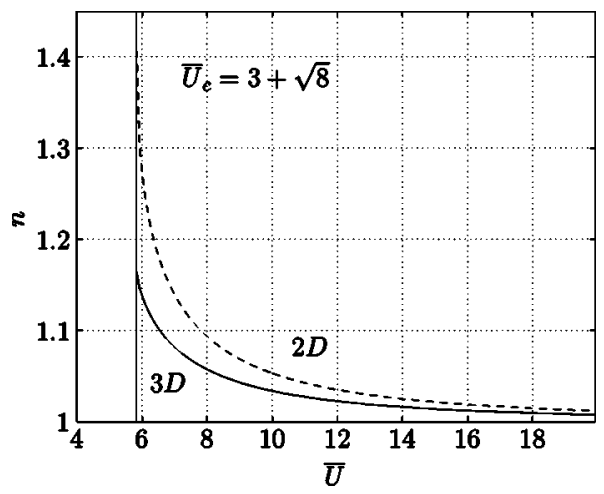

FIG. 10. Total density $n$ at $T=0$ as a function of interaction strength $\bar{U}=U / z t$ for the first Mott lobe in two (dashed line) and three dimensions (solid line) when including fluctuations. The density approaches a finite value different from 1, when approaching $U_{c}$.

This is very useful indeed since the correlator $\left\langle\Phi_{\mathbf{k}, n}^{*} \Phi_{\mathbf{k}, n}\right\rangle$ $=-G\left(\mathbf{k}, i \omega_{n}\right)$. At zero temperature the retarded Green's function can be written as

$$
-\frac{1}{\hbar} G(\mathbf{k}, \omega)=\frac{Z_{\mathbf{k}}}{-\hbar \omega+\epsilon_{\mathbf{k}}^{q p}}+\frac{1-Z_{\mathbf{k}}}{-\hbar \omega+\epsilon_{\mathbf{k}}^{q h}}+\frac{1}{\epsilon_{\mathbf{k}}},
$$

where the wave-function renormalization factor is

$$
Z_{\mathbf{k}}=\frac{U\left(1+2 \alpha^{\prime}\right)-\epsilon_{\mathbf{k}}+\sqrt{U^{2}-2 U \epsilon_{\mathbf{k}}\left(1+2 \alpha^{\prime}\right)+\epsilon_{\mathbf{k}}^{2}}}{2 \sqrt{U^{2}-2 U \epsilon_{\mathbf{k}}\left(1+2 \alpha^{\prime}\right)+\epsilon_{\mathbf{k}}^{2}}}
$$

and

$$
\begin{aligned}
\epsilon_{\mathbf{k}}^{q p, q h}= & -\mu+\frac{U}{2}\left(2 \alpha^{\prime}-1\right)-\frac{\epsilon_{\mathbf{k}}}{2} \\
& \pm \frac{1}{2} \sqrt{\epsilon_{\mathbf{k}}^{2}-\left(4 \alpha^{\prime}+2\right) U \epsilon_{\mathbf{k}}+U^{2}} .
\end{aligned}
$$

Note that $Z_{\mathbf{k}}$ is always positive and in the limit where $U$ $\rightarrow \infty$ we have that $Z_{\mathbf{k}} \rightarrow\left(1+\alpha^{\prime}\right)$. The quasiparticle dispersion $\epsilon_{\mathbf{k}}^{q p}$ is always greater than or equal to zero and $\epsilon_{\mathbf{k}}^{q h}$ is always smaller than or equal to zero. Because of this only the quasiholes give a contribution to the total density at zero temperature. The density can be calculated from

$$
\begin{aligned}
n & =\frac{1}{N_{s} \hbar \beta} \sum_{\mathbf{k}, n}\left\langle a_{\mathbf{k}, n}^{*} a_{\mathbf{k}, n}\right\rangle=\frac{1}{N_{s} \hbar \beta} \sum_{\mathbf{k}, n}\left\{-G\left(\mathbf{k}, \omega_{n}\right)-\frac{\hbar}{\epsilon_{\mathbf{k}}}\right\} \\
& \beta \rightarrow \infty \\
& =\frac{1}{N_{s}} \sum_{\mathbf{k}}\left(Z_{\mathbf{k}}-1\right) \stackrel{U \rightarrow \infty}{=} \alpha^{\prime} .
\end{aligned}
$$

If we expand the square-root denominator of $Z$ for small $\mathbf{k}$ we see that it behaves as $1 / \mathbf{k}$, therefore in two and three dimensions we expect the integration over $\mathbf{k}$ to converge. In Fig. 10 we have plotted the density for $\alpha^{\prime}=1$ as given by the equation above. We see that the density quickly converges to 1 , but near the tip of the Mott lobe in all dimensions it 
deviates significantly from 1 . This result is somewhat unexpected [9] and may be due to the breakdown of the Gaussian approximation near the quantum phase transition. A more detailed study of the fluctuations is beyond the scope of the present paper and is therefore left to future work.

\section{CONCLUSIONS}

In summary, we have applied the slave-boson formalism to the Bose-Hubbard model, which enabled us to analytically describe the physics of this model at nonzero temperatures. We have reproduced the known zero-temperature results and we have computed the critical temperature for the superfluidnormal phase transition. The crossover from a Mott insulator to a normal phase has also been quantified. We have shown how thermal fluctuations introduce additional dispersion modes associated with paired quasiparticles-quasiholes propagating through the system. We have also considered density fluctuations induced by the creation of quasiparticlequasihole pairs. These fluctuations do not average out to zero in the Gaussian approximation.

\section{ACKNOWLEDGMENTS}

This work is part of the research program of the "Stichting voor Fundamenteel Onderzoek der Materie (FOM)," which is financially supported by the "Nederlandse Organisatie voor Wetenschaplijk Onderzoek (NWO)."

\section{APPENDIX A: HIGHER-ORDER TERMS}

If we also want to calculate quantities like the superfluid density, we have to calculate the effective action up to fourth order. One way to do this is by going to higher order in the interaction part. Here we follow a slightly different strategy. Because we are only interested in the mean-field theory, it suffices to just consider $\Phi_{\mathbf{0}, 0}$ terms. The effective action for $\Phi_{0,0}$ is found from

$$
\begin{aligned}
Z= & \int \frac{d\left[\Phi_{0,0}^{*}\right] d\left[\Phi_{\mathbf{0}, 0}\right]}{\hbar \beta} \int \prod_{\alpha, \mathbf{k}, n} \frac{d\left[\left(a^{\alpha}\right)_{\mathbf{k}, n}^{*}\right] d\left[a_{\mathbf{k}, n}^{\alpha}\right]}{\hbar \beta} \\
& \times \exp \left(-\frac{1}{\hbar} S\right),
\end{aligned}
$$

where from Eq. (6) we have

$$
S=i N_{s} \hbar \beta \lambda+\epsilon_{\mathbf{0}}\left|\Phi_{\mathbf{0}, 0}\right|^{2}+\sum_{\alpha \beta} \sum_{\mathbf{k}, n}\left(a_{\mathbf{k}, n}^{\alpha}\right)^{*} M^{\alpha \beta} a_{\mathbf{k}, n}^{\beta} .
$$

Note, however, that now the matrix $M$ is only block diagonal and it contains off-diagonal terms proportional to $\Phi_{\mathbf{0}, 0}$. When we take the determinant of that matrix, you get automatically all powers in $\Phi_{\mathbf{0}, 0}$. This can be made more explicit by looking at the block structure of the matrix which is

$$
M=\left(\begin{array}{cccc}
B_{0} & & & \\
& B_{2} & & \\
& & B_{4} & \\
& & & \ldots
\end{array}\right),
$$

where

$$
B_{\alpha}=\left(\begin{array}{cc}
\chi_{\alpha} & \frac{\sqrt{\alpha+1}}{\sqrt{N_{s} \hbar \beta}} \epsilon_{0} \Phi_{0,0} \\
\frac{\sqrt{\alpha+1}}{\sqrt{N_{s} \hbar \beta}} \epsilon_{0} \Phi_{0,0}^{*} & \chi_{\alpha+1}
\end{array}\right)
$$

with $\chi_{\alpha}=-i \hbar \omega_{n}-i \lambda-\alpha \mu+\alpha(\alpha-1) U / 2$. The slave bosons can be integrated out with the result

$$
\begin{aligned}
Z= & \int \frac{d\left[\Phi_{\mathbf{0}, 0}^{*}\right] d\left[\Phi_{\mathbf{0}, 0}\right]}{\hbar \beta} \exp \left\{-\frac{1}{\hbar}\left(i N_{s} \hbar \beta \lambda+\boldsymbol{\epsilon}_{\mathbf{0}}\left|\Phi_{\mathbf{0}, 0}\right|^{2}\right)\right\} \\
& \times \exp \left\{-\sum_{\mathbf{k}, n} \ln [\operatorname{det} \beta M]\right\} .
\end{aligned}
$$

The determinant can be calculated up to fourth order in $\Phi_{0,0}$ as

$$
\begin{aligned}
\operatorname{det} \beta M= & \left(\prod_{\alpha} \beta \chi_{\alpha}\right)\left(1+\sum_{\alpha} \frac{\epsilon_{\mathbf{0}}^{2}}{N_{s} \hbar \beta}\left|\Phi_{\mathbf{0}, 0}\right|^{2} \frac{(\alpha+1)}{\chi_{\alpha} \chi_{\alpha+1}}\right. \\
& \left.+\sum_{\alpha} \sum_{|\alpha-\beta| \geqslant 2} \frac{\epsilon_{\mathbf{0}^{4}}}{\left(N_{s} \hbar \beta\right)^{4}}\left|\Phi_{\mathbf{0}, 0}\right|^{4} \frac{(\alpha+1)(\beta+1)}{\chi_{\alpha} \chi_{\alpha+1} \chi_{\beta} \chi_{\beta+1}}\right) .
\end{aligned}
$$

For small $\Phi_{0,0}$ we can expand the logarithm in Eq. (A4) by using the Taylor expansion

$$
\ln \left\{1-\alpha x^{2}+\gamma x^{4}\right\}=-\alpha x^{2}+\frac{1}{4}\left(-2 \alpha^{2}+4 \gamma\right) x^{4}+O\left(x^{5}\right) .
$$

Combining the latter equation with Eq. (A4), we also recover that the second-order term in the effective action for $\Phi_{0,0}$ is given by

$$
\begin{aligned}
& \left(\epsilon_{\mathbf{0}}-\hbar \sum_{\mathbf{k}, n} \sum_{\alpha} \frac{\epsilon_{\mathbf{0}}^{2}}{N_{s} \hbar \beta} \frac{(\alpha+1)}{\chi_{\alpha} \chi_{\alpha+1}}\right)\left|\Phi_{\mathbf{0}, 0}\right|^{2} \\
& =\left(\epsilon_{\mathbf{0}}+\epsilon_{\mathbf{0}}^{2} \frac{n^{\alpha}-n^{\alpha+1}}{-\mu+\alpha U}\right)\left|\Phi_{\mathbf{0}, 0}\right|^{2}=-\hbar G^{-1}(\mathbf{0}, 0)\left|\Phi_{\mathbf{0}, 0}\right|^{2} .
\end{aligned}
$$

We determine the effective action to fourth order in the case of the first four slave bosons. Using the above we can readily verify that 


$$
\begin{aligned}
-S^{\mathrm{eff}} / \hbar= & -\frac{1}{\hbar}\left\{\boldsymbol{\epsilon}_{\mathbf{0}}\left|\Phi_{\mathbf{0}, 0}\right| 2+i N_{s} \hbar \beta \lambda-\sum_{j=0}^{3} \ln \beta \chi_{j}\right. \\
& -\ln \left[1-\left(\frac{\boldsymbol{\epsilon}_{\mathbf{0}}}{N \hbar \beta}\right)^{2}\left(\frac{3}{\chi_{3} \chi_{2}}+\frac{2}{\chi_{2} \chi_{1}}+\frac{1}{\chi_{1} \chi_{0}}\right)\left|\Phi_{\mathbf{0}, 0}\right|^{2}\right. \\
& \left.\left.+\left(\frac{\epsilon_{\mathbf{0}}}{N_{s} \hbar \beta}\right)^{4} \frac{3}{\chi_{0} \chi_{1} \chi_{2} \chi_{3}}\left|\Phi_{\mathbf{0}, 0}\right|^{4}\right]\right\} .
\end{aligned}
$$$$
\begin{aligned}
a_{4}= & -\left(\frac{\epsilon_{\mathbf{0}}}{2 N_{s}^{2} \hbar \beta}\right)\left\{\frac{9}{(2 \bar{U}-\bar{\mu})^{2}}\left[3 n^{3}\left(1-n^{3}\right)+2 n^{2}\left(1-n^{2}\right)\right]+\frac{18}{(2 \bar{U}-\bar{\mu})^{3}}\left(n^{3}-n^{2}\right)+\frac{4}{(\bar{U}-\bar{\mu})^{2}}\left[2 n^{2}\left(1-n^{2}\right)+n^{1}\left(1-n^{1}\right)\right]\right. \\
& +\frac{8}{(\bar{U}-\bar{\mu})^{3}}\left(n^{2}-n^{1}\right)+\frac{1}{(\bar{\mu})^{2}}\left[n^{0}\left(1-n^{0}\right)+n^{1}\left(1-n^{1}\right)\right]+\frac{2}{\bar{\mu}^{3}}\left(n^{0}-n^{1}\right)+\frac{4}{(\bar{U}-2 \bar{\mu}) \bar{\mu}^{2}} n^{0}-\frac{4}{(\bar{U}-2 \bar{\mu})(\bar{U}-\bar{\mu})^{2}} n^{2} \\
& +\frac{4}{(\bar{U}-\bar{\mu}) \bar{\mu}} n^{1}\left(1-n^{1}\right)-\frac{4 \bar{U}}{(\bar{U}-\bar{\mu})^{2} \bar{\mu}^{2}} n^{1}-\frac{12}{(3 \bar{U}-2 \bar{\mu})(2 \bar{U}-\bar{\mu})^{2}} n^{3}-\frac{12}{\left(2 \bar{U}^{2}-3 \bar{U} \bar{\mu}+\bar{\mu}^{2}\right)} 2 n^{2}\left(1-n^{2}\right) \\
& \left.-\frac{12 \bar{U}}{\left(2 \bar{U}^{2}-3 \bar{U} \bar{\mu}+\bar{\mu}^{2}\right)^{2}} n^{2}+\frac{12}{(3 \bar{U}-2 \bar{\mu})(\bar{U}-\bar{\mu})^{2}} n^{1}\right\} .
\end{aligned}
$$

From this we find that $a_{4}$ in the case of four slave bosons is given by

$$
a_{4}=\frac{\hbar}{4}\left(\frac{\epsilon_{\mathbf{0}}}{\sqrt{N_{s} \hbar \beta}}\right)^{4} \sum_{\mathbf{k}, n}\left[-2\left(\sum_{\alpha=0}^{3} \frac{(\alpha+1)}{\chi_{\alpha} \chi_{\alpha+1}}\right)^{2}+\frac{12}{\chi_{0} \chi_{1} \chi_{2} \chi_{3}}\right],
$$

or explicitly,
Note that in the zero-temperature limit for the first Mott lobe, when the slave-boson occupation numbers are proportional to a Kronecker delta, this result coincides exactly with the one previously derived in standard perturbation theory (cf. Ref. [4]).

\section{APPENDIX B: DENSITY CALCULATIONS}

In this appendix we demonstrate for the noninteracting case the equivalence of the calculation of the total particle density through the thermodynamic relation $N=-\partial \Omega / \partial \mu$ and through the use of source currents that couple to the atomic fields. We consider a system of noninteracting bosons described by creation and annihilation fields $a_{i}^{*}(\tau)$ and $a_{i}(\tau)$ on a lattice. First we calculate the generating functional $Z\left[J^{*}, J\right]$ for this system,

$$
\begin{aligned}
Z\left[J^{*}, J\right]= & \int d\left[a^{*}\right] d[a] \exp \left\{-\frac{1}{\hbar} S_{0}\left[a^{*}, a\right]\right. \\
& \left.+\frac{1}{\hbar} \int d \tau \sum_{i j} a_{i}^{*} t_{i j} a_{j}+\int d \tau \sum_{i}\left(J_{i}^{*} a_{i}+a_{i}^{*} J_{i}\right)\right\} .
\end{aligned}
$$

In this equation $S_{0}$ is the on-site action, which in frequencymomentum representation typically looks like

$$
S_{0}\left[a^{*}, a\right]=\sum_{\mathbf{k}, n} a_{\mathbf{k}, n}^{*}\left(-i \hbar \omega_{n}-\mu\right) a_{\mathbf{k}, n} .
$$

The hopping term is decoupled by means of a HubbardStratonovich transformation, i.e., we add the following complete square to the action:

$$
\sum_{i j}\left(a_{i}^{*}-\Phi_{i}^{*}+\hbar \sum_{j^{\prime}} t_{i j^{\prime}}^{-1} J_{j^{\prime}}^{*}\right) t_{i j}\left(a_{j}-\Phi_{j}+\hbar \sum_{j^{\prime \prime}} t_{i j^{\prime \prime}}^{-1} J_{j^{\prime \prime}}\right) .
$$

The atomic fields $a^{*}, a$ can now be integrated out. Going through the straightforward algebra one arrives at the following expression for the generating functional:

$$
\begin{aligned}
Z\left[J^{*}, J\right]= & \int d\left[\Phi^{*}\right] d[\Phi] \exp \left\{\sum_{\mathbf{k}, n} \Phi_{\mathbf{k}, n}^{*} G^{-1}\left(\mathbf{k}, i \omega_{n}\right) \Phi_{\mathbf{k}, n}\right. \\
& \left.+J_{\mathbf{k}, n}^{*} \Phi_{\mathbf{k}, n}+J_{\mathbf{k}, n} \Phi_{\mathbf{k}, n}^{*}-\hbar \frac{J_{\mathbf{k}, n} J_{\mathbf{k}, n}^{*}}{\epsilon_{\mathbf{k}}}\right\}
\end{aligned}
$$

where $-\hbar G^{-1}\left(\mathbf{k}, i \omega_{n}\right)=\epsilon_{\mathbf{k}}-\epsilon_{\mathbf{k}}^{2}\left(-i \hbar \omega_{n}-\mu\right)^{-1}$. The total density may be calculated from this expression by first calculating the correlator $\left\langle a_{\mathbf{k}, n}^{*} a_{\mathbf{k}, n}\right\rangle$ through functional differentiation with respect to the source currents $J$, and then to sum over all momenta and Matsubara frequencies. We have for the first step

$$
\begin{aligned}
\left\langle a_{\mathbf{k}, n}^{*} a_{\mathbf{k}, n}\right\rangle & =\left.\frac{1}{Z[0,0]} \frac{\delta^{2}}{\delta J_{\mathbf{k}, n}^{*} \delta J_{\mathbf{k}, n}} Z\left[J^{*}, J\right]\right|_{J *, J=0} \\
& =\frac{\hbar}{-i \hbar \omega_{n}-\mu-\epsilon_{\mathbf{k}}} .
\end{aligned}
$$

We see that there is a pole here at $i \hbar \omega_{n}=-\epsilon_{\mathbf{k}}-\mu$. The density now can be calculated from $n$ $=\left(1 / N_{s} \hbar \beta\right) \Sigma_{\mathbf{k}, n}\left\langle a_{\mathbf{k}, n}^{*} a_{\mathbf{k}, n}\right\rangle$. This is the expected result. 
On the other hand, we can also calculate the density from the thermodynamic potential $\Omega$, by using the relation $N$ $=-\partial \Omega / \partial \mu$ where $N$ is the total number of particles. Doing that for this case we use that

$$
\Omega=\frac{1}{\beta} \sum_{\mathbf{k}, n}\left\{\ln \left[\beta\left(-i \hbar \omega_{n}-\mu\right)\right]+\ln \left[-\hbar \beta G^{-1}\left(\mathbf{k}, i \omega_{n}\right)\right]\right\}
$$

and obtain

$$
\begin{aligned}
n= & -\frac{1}{N_{s}} \frac{\partial \Omega}{\partial \mu}=\frac{1}{N_{s} \hbar \beta} \sum_{\mathbf{k}, n}\left\{\frac{\hbar}{-i \hbar \omega_{n}-\mu}\right. \\
& \left.+\frac{\hbar}{-i \hbar \omega_{n}-\mu-\epsilon_{\mathbf{k}}} \frac{\epsilon_{\mathbf{k}}}{-i \hbar \omega_{n}-\mu}\right\} .
\end{aligned}
$$

When doing the sum over Matsubara frequencies the pole at $i \hbar \omega_{n}=-\mu$ in the first term in the right-hand side is canceled by the second term and only the other pole at $i \hbar \omega_{n}=-\epsilon_{\mathbf{k}}$ $-\mu$ gives a contribution. This shows the equivalence of both methods.
[1] M.P.A. Fisher, P.B. Weichman, G. Grinstein, and D.S. Fisher, Phys. Rev. B 40, 546 (1989).

[2] D. Jaksch, C. Bruder, J.I. Cirac, C.W. Gardiner, and P. Zoller, Phys. Rev. Lett. 81, 3108 (1998).

[3] K. Sheshadri, H.R. Krishnamurthy, R. Pandit, and T.V. Ramakrishnan, Europhys. Lett. 22, 257 (1993).

[4] D. van Oosten, P. van der Straten, and H.T.C. Stoof, Phys. Rev. A 63, 053601 (2001).

[5] D. van Oosten, P. van der Straten, and H.T.C. Stoof, Phys. Rev. A 67, 033606 (2003).

[6] R. Roth and K. Burnett, Phys. Rev. A 67, 031602 (2003).

[7] M. Greiner, O. Mandel, T. Esslinger, T.W. Hänsch, and I. Bloch, Nature (London) 415, 39 (2002).

[8] W. Zwerger, J. Opt. B 5, s9 (2003).

[9] S. Sachdev, Quantum Phase Transitions (Cambridge University Press, Cambridge, 2001).

[10] G. Kotliar and A.E. Ruckenstein, Phys. Rev. Lett. 57, 1362 (1986).

[11] K. Ziegler, Europhys. Lett. 23, 463 (1993).

[12] R. Frésard, e-print cond-mat/9405053.

[13] J.W. Negele and H. Orland, Quantum Many-Particle Systems
(Addison-Wesley, Redwood City, 1988).

[14] H.T.C. Stoof, in Coherent Atomic Matter Waves, edited by R. Kaiser and F. David (Springer, Berlin, 2001), p. 219.

[15] A remedy for this discrepancy may be envisioned in allowing the right-hand side of Eq. (34) to deviate sligthly from 1 . We do not explore this possibility here.

[16] We have also studied the case where the $\left(n^{3}-n^{2}\right)$ term is included with $n^{3}$ put equal to 0 . This leads to the undesirable feature that $T_{\mathrm{c}} \rightarrow 0$ for $U \rightarrow 0$. Including this term, which is associated with processes involving triply occupied sites, probably is inconsistent with allowing a maximum occupancy of 2 .

[17] For instance, at $U=0$, the three-slave-boson approach can only result in $n^{0}=n^{1}=n^{2}=1 / 3$, whereas the exact solution for four bosons on a four-site lattice gives $n^{0}=0.35, n^{1}=0.38$, $n^{2}=0.20, n^{3}=0.059$, and $n^{4}=0.008$ at $\beta=1$ [19].

[18] In particular, for $\bar{U}=4$ we find at $T_{\mathrm{c}}$ that $n^{0}=0.125, n^{1}$ $=0.751, n^{2}=0.122$, and $n^{3}=0.0018$, whereas the three-slaveboson result is $n^{0}=0.111, n^{1}=0.778$, and $n^{2}=0.111$.

[19] G.G. Batrouni and R.T. Scalettar, Phys. Rev. B 46, 9051 (1992). 